\title{
Observations on the reaction of the intestinal mucosa to bacterial challenge
}

\author{
R. KENWORTHY
}

From the Unilever Research Laboratory, Colworth House, Sharnbrook, Bedford

In all species of animals, including man, the epithelium of the gastrointestinal tract is in constant contact with microorganisms. Furthermore, according to Smith (1965), the flora of the alimentary tract in all the homeothermic animals examined by him consisted principally of the same types of organism. However, numbers varied greatly and were in no small measure related to the diet and to the frequency of feeding. Smith (1965) also referred to the difficulty which can be experienced in establishing microorganisms in the alimentary tracts of animals even after administration of extremely large numbers, but some success can be achieved by a radical alteration in dietary formulation. Similarly, attempts to establish pathogens, with or without subsequent reproduction or production of enteric disease, have often given variable results except in very young animals deprived of colostrum (see review by Kenworthy, 1967) or where some other special experimental conditions were imposed (Freter, 1955; Cooper, 1959; Ashburner and Mushin, 1962; Takeuchi, Sprinz, LaBrec, and Formal, 1965). Thus in view of these observations, and the anatomical and physiological differences which occur between the intestines of germ-free and conventional animals of the same species (see review by Gordon, 1968) the intestinal flora must normally be regarded as a stable contributor to intestinal physiology and function. Furthermore, under conditions of health and after a variable period of adaptation in infancy dependent upon the species of animal under consideration, the mucosa must be regarded as conditioned to resist the continual challenge imposed by the bacterial load in the intestinal lumen. Under conditions of disease, however, microorganisms which are not normally regarded as indigenous to the tract are established and proliferate, or if indigenous they are normally present in low numbers only. At such a time, the mucosa has to readapt to the new challenge.

As a contribution to the further understanding of the physiology and pathology of the gastrointestinal tract it is thus of paramount importance to attempt to understand how the mucosal barrier operates $\vec{\omega}$ under normal conditions, and also the manner in which it can respond to a pathogen; this paperic considers some of these mechanisms. The epithelium '́r and its associated 'secretions' are examined first, $\rightarrow$ ' followed by a discussion of the response of the $\vec{\omega}$ lamina propria. This sequence is undertaken for convenience, and does not necessarily represent the priorities of these tissues in the defence response.Finally, the process of villous fusion under the influence of microbial challenge is examined and? discussed; all of the foregoing are considered with special reference to the pig.

The epithelium lining the gastrointestinal tract of monogastric animals is classically columnar,; although under certain circumstances foreshortenigig of the cells may occur (Sprinz, Kundel, Dammin, Horewitz, Schneider, and Formal, 1962) and they may become cuboidal (Kenworthy, 1970). There have been many publications describing the epithelial ${ }_{\Omega}^{\Phi}$ cell on the villus in its absorptive capacity, also the $\vec{F}$ epithelium of the crypts has frequently been identi-윽 fied as a generative compartment for replacement of cells lost from the extrusion zones. More pertinento to this discussion, however, is the intestine as a'secretory' organ, since intestinal 'secretions' contain! antibodies which appear to be important in protect-음 ing the epithelium against microbial activity in the intestinal lumen under conditions of health, as wello as against true pathogens (Davies, 1922; Burrows, Elliott, and Havens, 1947; Burrows and Havens, 1948; Freter, 1965; Berger, Ainbender, Hodes, $D$ Zepp, and Hevizy, 1967; Felsenfeld, Greer, and으. Felsenfeld, 1967; Porter, Noakes, and Allen, 1970). $\bar{N}$ However, it is not the intention here to review the literature concerning coproantibody or intestinal $N$ secretory immunoglobulins. It suffices to emphasize that the available evidence, both direct and indirect indicates that the two terms are synonymous, thato they refer to a local immune response in the gut $\frac{\leftarrow}{\Phi}$ which is largely, if not entirely, independent of ? circulating antibody.

Of the 'secretory' immunoglobulins with anti-음 
microbial antibody, IgA is the more important, qualitatively and quantitatively, under most circumstances. Further, $\operatorname{IgA}$ is synthesized by plasma cells in the lamina propria (Crabbé, Carbonara, and Heremans, 1965) and concentrated in and between epithelial cells in the lower two-thirds of the crypts of Lieberkuhn, the intracellular locus being almost entirely in the apical cytoplasm (Gelzayd, Kraft, and Fitch, 1967; Gelzayd, Kraft, and Krisner, 1968; Allen and Porter, 1970). (The significance of these observations will become apparent when the response of the epithelium and lamina propria to virulent pathogens is discussed.) Since IgA is produced by plasma cells in the intestinal lamina propria, and these cells are present in small numbers only in the germ-free animal (Crabbé, Bazin, Eyssen, and Heremans, 1968; Kenworthy, 1970), it is hardly surprising that we could not demonstrate $\operatorname{IgA}$ in the epithelium of germ-free pigs 31 days old; however, in gnotobiotes of the same age infected with viable Escherichia coli for four days, there were early signs of IgA 'secretion' in that a few crypt epithelial cells showed specific fluorescence in the supranuclear cytoplasm. Ten days after infection the picture resembled that found in conventional animals. The importance of this 'first line defence' mechanism to microbes (Thomasi, 1967) in the gut was thus again implied, since Porter and Kenworthy (1970) were able to show only fractions of conventional circulatory levels of IgA, IgM, or IgG in gnotobiotic pigs, even three weeks after infection with $E$. coli.

The bacterial antigen(s) mainly involved in stimulating the secretory IgA response have not yet been totally identified. Freter (1965) has considered this aspect of the subject relative to cholera. He found in his experimental models an excellent cross protective effect of antibody against the heat-stable group and type antigens of two strains of Vibrio cholerae. Porter et al (1970) have described experiments using pigs with Thiry-Vella loops, in which they recorded $\operatorname{IgA}$ as the major intestinal antibody to endotoxin of $E$. coli 0141 , although IgM and IgG were also present. This observation confirms the significance of secretory IgA as antibacterial antibody directed at least in part against the heat-stable $O$ somatic antigen. It is of additional interest because, although no attempt was made to determine whether the serotype against which antibody activity was assayed was part of the normal microflora of the experimental pigs, the particular organism used is a porcine pathogen. Therefore, even if present in the experimental animals, it would probably only occur in small numbers. Within the various genera and species of the Enterobacteriaceae there is much sharing of the $\mathbf{O}$ somatic antigens. Thus it appears from all these observations that some protection could be readily available to certain microorganisms which are not normally present in the intestinal flora of any particular animal or species of animal, but which may populate it in a disease state. The same argument would be even more applicable to those diseased states which are associated with the proliferation of microorganisms normally endemic to the environment of the animal concerned, but in small numbers only. It remains to be shown whether IgA or some other immunoglobulin secreted into the intestine has activity against other microbial antigens such as the ' $\mathrm{K}$ ' antigens of $E$. coli, since speculation has been made that at least one of these may have a biological attribute important in pathogenesis (Sojka, 1965). It is pertinent here to point out that Tomasi (1967) has referred to a possible relationship between secretory IgA and IgM, and Brandtzaeg (1968) has suggested that a common secretory mechanism for both IgA and IgM may be operative in man. Support for these observations can be found in that with cases of ataxia telangiectasia, where there is an IgA deficiency, the protective role in external secretions is adopted by IgM (Stobo and Tomasi, 1967; Eidelman and Davis, 1968). Further, Allen and Porter (1970) have shown the presence of IgM in the cytoplasm of crypt epithelial cells of young pigs 15 days old.

Thus the mucosa of the intestine can be regarded as bathed in a fluid which contains $\operatorname{IgA}$ and $\operatorname{IgM}$; the evidence to date firmly indicates IgA as the most significant component as a bacterial antibody, but final conclusions will have to await definition of activity against the various members of the microflora and to the different antigens which the latter contain.

In the presence of virulent pathogens it is evident that the intestinal antibody is insufficient, since systemic invasion occurs. Studies by Takeuchi et al (1965), Takeuchi (1967), and Kenworthy (1970) have shown that in guinea pigs and pigs the epithelium can, under the conditions specified, sequester microorganisms which have penetrated the cells. In some cases the organisms appeared to have undergone digestion within lysosome-like bodies, but in others a membrane-bound vesicle formed a protective mechanism whereby the intact organism reached the lamina propria. Some of these studies have been carried out with germ-free animals whose microbial experience has been limited to challenge with the pathogen. They do show, however, that under the experimental conditions defined the epithelium can limit systemic invasion by live cells. It appears too from these studies that it is the virulence of the organism which in part determines the efficacy of this area of the defence response.

It is of interest to refer at this point to the observa- 
tions of Takeuchi et al (1965) and Takeuchi (1967) relative to the crypt cells in their experimentalmodels. In experimental bacillary dysentery, crypt epithelium was distinctly less affected by bacterial invasion than were cells on the villous surface; with Salmonella typhimurium infection, the crypt epithelium was free from bacterial invasion, though many organisms were present in the crypt lumens. Similar observations have been made in Cryptosporidium enteritis in guinea pigs (Jervis, Merrill, and Sprinz, 1966). Takeuchi et al (1965) have suggested explanations for the resistance to invasion by crypt cells, basing their hypotheses on anatomical considerations. In view of the work described relative to the locus of $\operatorname{IgA}$ in intestinal epithelium, it is possible to conjecture that crypt epithelium is protected by the greater concentration of secretory $\operatorname{IgA}$ which is likely to occur on its free border.

Changes in morphology of epithelial cells during the course of reaction to intestinal microorganisms do occur, but the opinion is that they are in the main consequent upon the inflammatory reaction in the lamina propria. The changes referred to may take the forms of reduction in cell volume, a cuboibal appearance, detachment from the underlying basement membrane, and intercellular separation up to the level of the tight junction. Takeuchi et al (1965) have described loss of cytoplasmic components and particularly severe regressive alterations in the microvilli and mitochondria in epithelial cells challenged by Shigella. There was loss of chromatin pattern from nuclei, nuclear swelling, and irregular configuration with occasional obliteration of nucleoli. In response to infection with a pathogenic serotype of $E$. coli, Kenworthy (1970) has referred to loss of nuclear staining affinity, nuclear swelling, and intense acidophilia of cytoplasm in crypt epithelium. In villous epithelium apparently dependent on the duration of the infection there was partial atrophy and infranuclear vacuolation of epithelial cells (Fig. 1), followed by further atrophic changes and creation of wide, intercellular spaces. Previous interdigitations of apposed cell walls then protruded as finger-like processes. Associated with cell atrophy were crenation of nuclei and of mitochondria. When atrophic changes occurred they were always associated with increased interdigitation at about the level of the tight junction. Further, in animals infected for longer periods of time, advanced lytic changes occurred (Fig. 2) which involved epithelium and lamina over variable lengths of the villi, but always included the tips.

Where live organisms have been used to infect animals, such as initially germ-free guinea pigs (Sprinz et al, 1962), preconditioned conventionally reared guinea pigs (Takeuchi et al, 1965; Takeuchi and Sprinz, 1967), and initially germ-free pigs 7 (Kenworthy, 1970), descriptions of the response of the lamina propria have been in close agreement. 은 Sprinz et al (1962) have considered the response of $\Rightarrow$ the germ-free guinea pig to oral bacterial challenge with $E$. coli and Shigella flexneri. The animals were? killed several days after infection had been intro- $\frac{\bar{\sigma}}{\bar{c}}$. duced and therefore presumably the initial acute $\frac{\rho}{\sigma}$

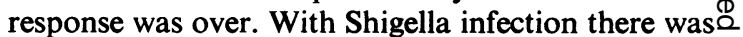
evidence of inflammatory oedema in the lamina propria, together with large amounts of Feulgen- $\overrightarrow{0}$ position pyknotic nuclear debris; the cellularresponse at the time of examination was almost $\vec{\omega}$ exclusively comprised of large macrophages. There? was distension of lymphatics and a marked venous? hyperaemia.

Takeuchi et al (1965), using Shigella as the infectiveć agent, have described an exudative inflammatory:reaction in the lamina propria which preceded altera- $\vec{\infty}_{\infty}$

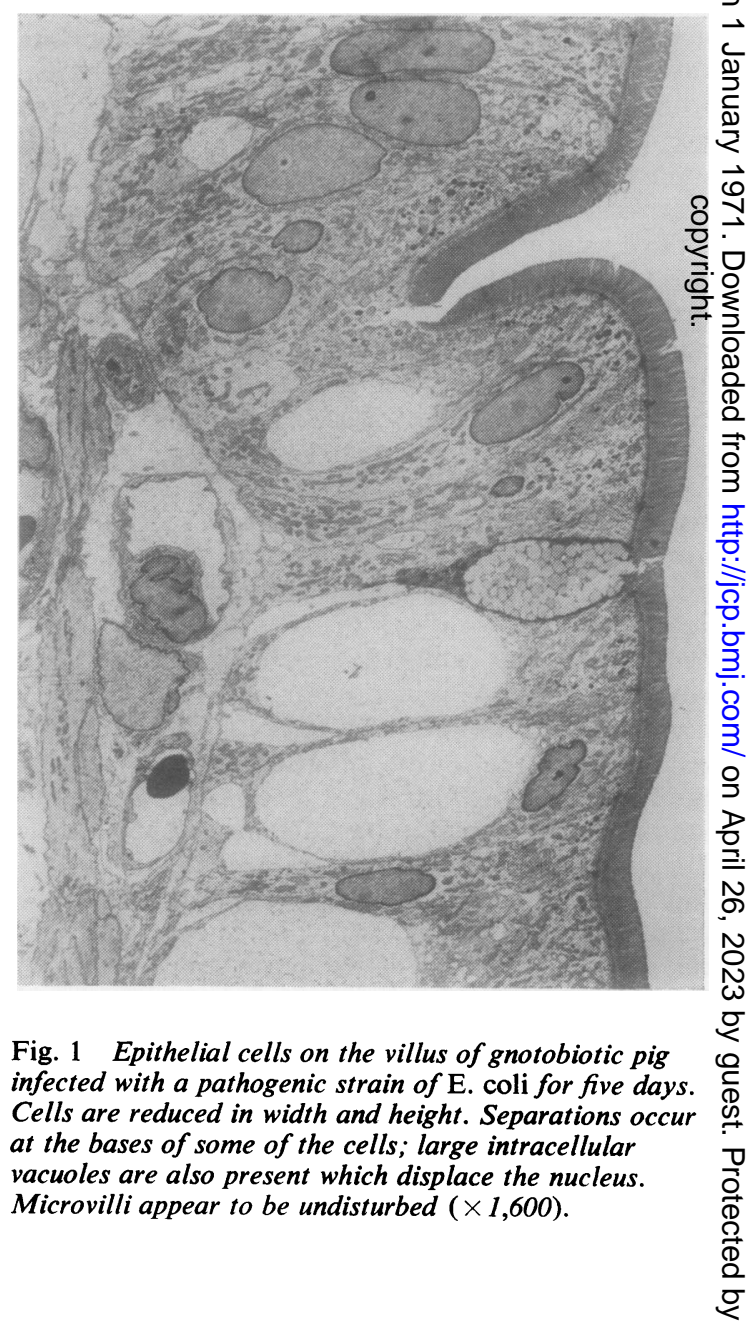




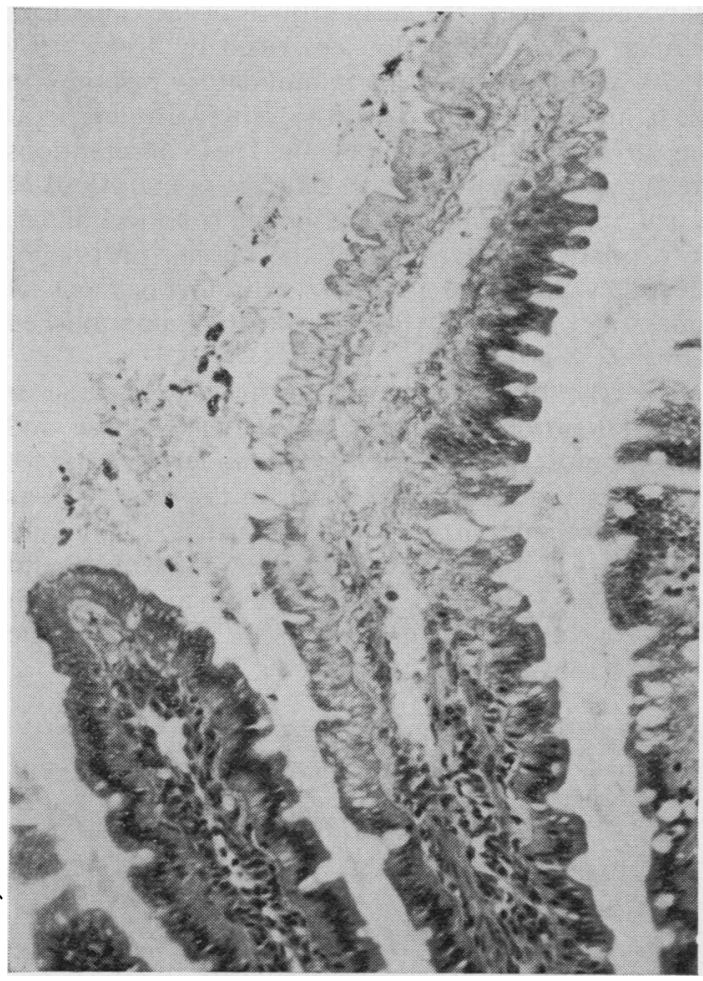

Fig. 2 Villus of gnotobiotic pig infected with pathogenic strain of $\mathrm{E}$. coli for 21 days. Extensive degenerative changes occur, involving over half of one side of the villus. Note also an area of focal degeneration sandwiched between two less affected areas $(H \& E \times 80)$.

tions in the villous epithelium. Leucocytes and macrophages participated in the response and packed the lamina. The authors have related this early response, which occurred not later than eight hours after infection, to the presence of a very few organisms in the epithelium.

Similarly, describing experimental infection of the preconditioned guinea pig with Salmonella typhimurium, Takeuchi and Sprinz (1967) have observed vascular changes in the lamina propria without alteration in the intracytoplasmic organelles of the epithelium. The changes in the lamina were those of a progressive inflammatory reaction, and there was an inflammatory cell exudate associated with increased transepithelial migration of these cells.

Kenworthy (1970) has described the morphological response of the intestines of gnotobiotic pigs to short-term or slightly more prolonged infection with a pathogenic strain of $E$. coli, and with minimal

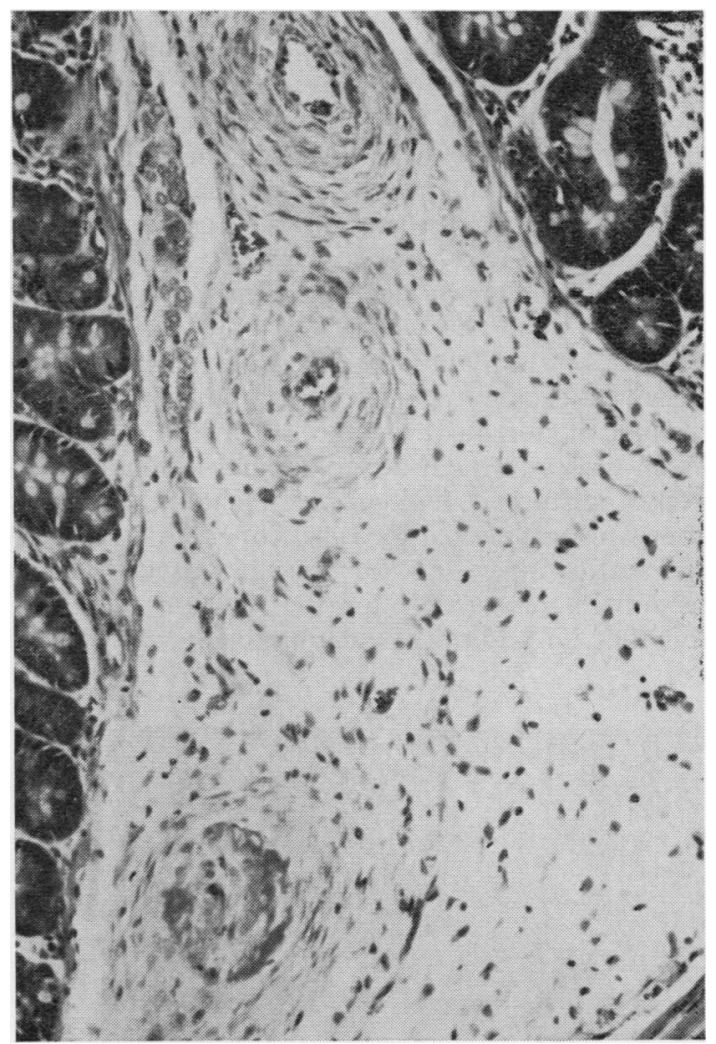

Fig. 3 Small vessels in submucosa of gnotobiotic pig infected with pathogenic strain of $\mathrm{E}$. coli for 21 days. The vessel at the left of the picture is totally occluded $(H \& E \times 80)$.

antigenic stimulation from any other source such as the diet. There was an exudative response in the lamina, with dilatation and hyperaemia of the villous capillaries; villous cores were infiltrated with polymorphonuclear leucocytes, and there was an increase in numbers of macrophages, lymphocytes, and plasma cells. Furthermore, the distribution of these cells was of great interest. Lymphocytes commonly entered the epithelial borders of villi, and although plasma cells were increased in number, they were never found above the lower third of the intercryptal lamina propria. Additionally, in animals which had been exposed to infection for several days degenerative changes occurred in the tunica media of arteries and arterioles of the submucosa, associated with extensive hyperplasia of the adventitial fibroblastic tissue. The lesion was so extensive on occasion as to occlude the lumen of the vessel (Fig. 3). The changes described occurred in the absence of any 
evidence to show that bacterial penetration of the gut had proceeded further than the epithelial cell, and in the absence of any marked changes in circulating antibody (Porter and Kenworthy, 1970). Furthermore, the morphological alterations in the epithelium were such as to suggest that they were in good measure dependent on the response in the lamina propria (Kenworthy, 1970), rather than influenced directly by the organisms in the lumen of the gut.

Some of the data considered here indicate that the initial exciting agent to the lamina propria is a product of microbial metabolism in the gut lumen which passes through the epithelium leaving it relatively undisturbed. It seems possible that the number of organisms detected by Takeuchi et al (1965) eight hours after infection with Shigella was too few to be entirely responsible for the reaction described, and that an additional exciting agent was a toxin

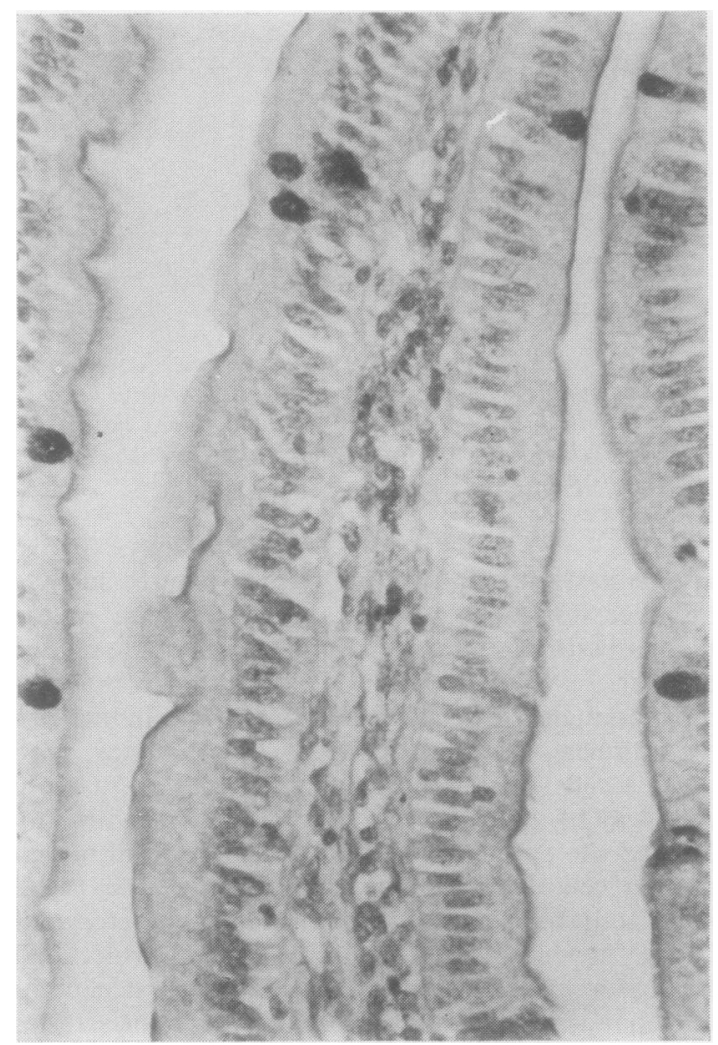

Fig. 4 Villus of gnotobiotic pig infected with pathogenic strain of $\mathrm{E}$. coli for six days. Blurring of the epithelial cell border is clearly shown $(P A S \times 200)$. elaborated during the early growth of the organism. To Similarly, in the infection described by Kenworthy (1970) the severity of the inflammatory response in the lamina is difficult to identify only with degenera- $\vec{F}$ ting organisms in epithelial cells. These observations $\stackrel{\vec{P}}{\rightarrow}$ are supported by studies on cell-free preparations of $E$. coli metabolism using the ligated loop technique; $\frac{\bar{\sigma}}{\bar{\sigma}}$ an exudative hyperaemia of the lamina propria of $\frac{\bar{D}}{\vec{\sigma}}$ the villus could be induced, with involvement of $\varrho$ inflammatory cells (R. Kenworthy, unpublished के data).

The reaction of the lamina propria to bacteria or $\stackrel{\overrightarrow{0}}{-}$ bacterial products of metabolism was intense and $\vec{\omega}$ acute in most of the experimental models referred to. The involvement of plasma cells in the response has already been referred to (Kenworthy, 1970), and $\%$ Crabbé et al (1965), Rubin, Fauci, Sleisenger, and ó Jeffries (1965), and Crandall, Cebra, and Crandall $-\overrightarrow{-}$ (1967) have shown that a unique feature of the $\underset{\infty}{\vec{\omega}}$

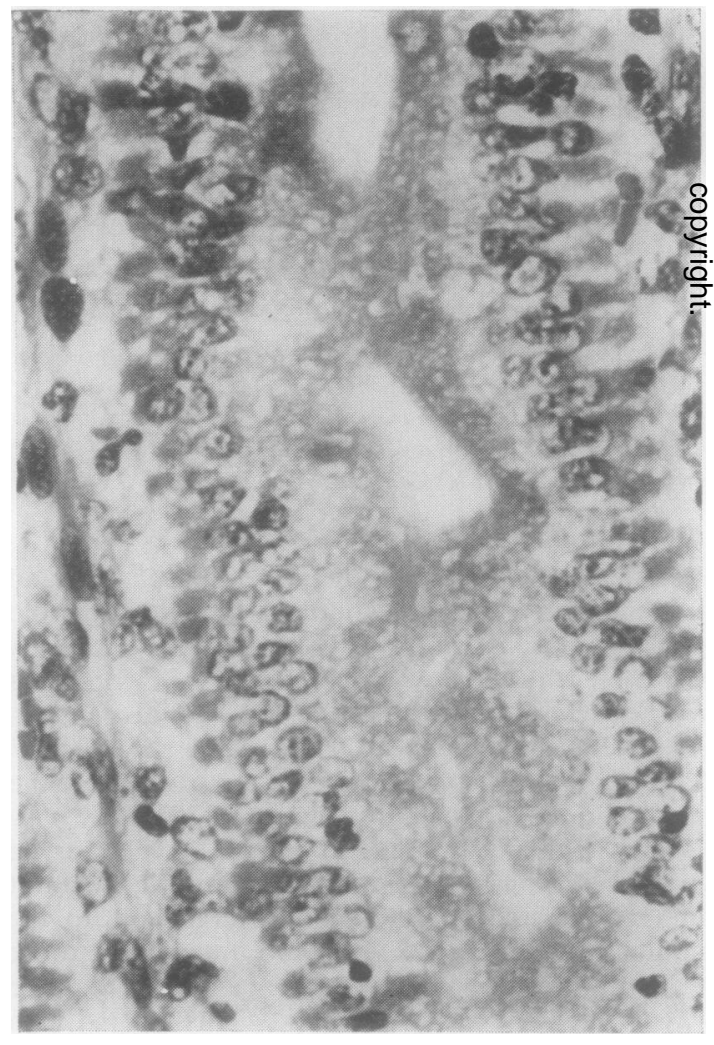

Fig. 5 Epithelial cells on apposed villi of gnotobiotic pig infected as described for Figure 4. Areas of apparent union between the villi are shown $(H \& E$ $\times 320$ ). 
intestinal plasma cell system in normal humans and rabbits is that most of the plasma cells synthesize IgA. Furthermore Crabbé et al (1968) have quantified the differences between conventional and germfree mice in terms of plasma cell populations in the gut. In the germ-free mice, IgA cell populations were one tenth or less of the conventional values. Thus a direct causal relationship between the presence of a living microbial flora and the development of cells synthesizing IgA in the intestinal mucosa was established, and correlates with observations already referred to in connexion with secretory $\operatorname{IgA}$ and microbial challenge. However, the experiments described by Takeuchi et al (1965) and Kenworthy (1970) have also served to emphasize the participation of phagocytic mechanisms in the defence response and this is an area which will merit further study. The polymorphonuclear leucocyte and the macrophage were prominent in the reactions described, and Kenworthy (1970) has drawn attention to the disposition of these cells within the villus of the pig, and also to the complexes which macrophages in the lower lamina form with plasma cells and eosinophils. The suggestion has been made that macrophages may convey antigen from the villous tip to the lower lamina, ie, to the region in which the plasma cells are mainly located.

Finally, it is pertinent to consider the significance of these inflammatory reactions as determinants of villous morphology. The acute phase with its inflam-

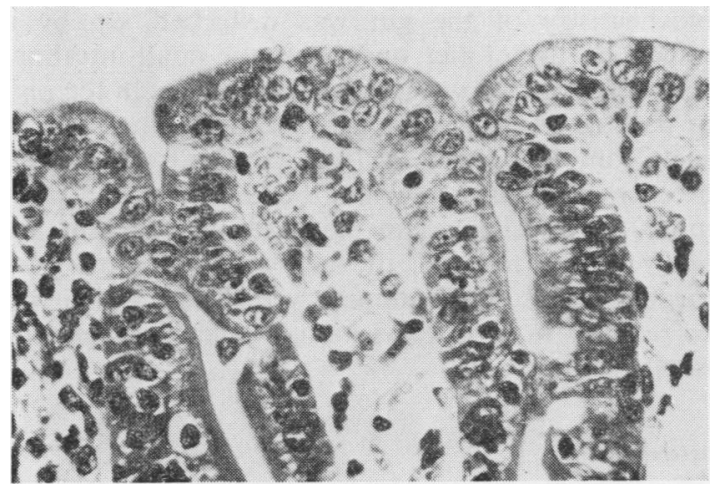

Fig. 6 Apparent insertion of epithelium of one villus into another, thereby creating a 'bridge' between two adjacent villi $(H \& E \times 140)$.

Fig. 7 Tip of a villus to show amount of interepithelial cell separation which can occur by removal of one cell $(H \& E \times 320)$. matory exudate swells and foreshortens the villi, distorts the topography of the connective tissue of the villous core, and lengthens the crypts (Takeuchi and Sprinz, 1967; Kenworthy, 1970). Intervillous fusion also occurs, and this appears to arise where two adjacent swollen villi come into apposition. A possible hypothesis is that two epithelial surfaces may merge over a limited extent. Below the point of fusion the surfaces become independent again, and a true pocket or channel lined with epithelium is thus created. The author has never at any time seen any fibroblastic proliferation associated with the phenomenon of fusion, but it is common to see blurring of the luminal surface of epithelial cells on the walls of the villi, with loss of definition of the brush border; by light microscopy the effect is to combine the brush border and the luminal cell membrane together with its immediately associated cytoplasm into a homogenous structure of foamy appearance (Fig. 4). The structure so formed is faintly acidophilic and faintly PAS positive, but does not give the impression that it is associated with increased production of mucin. Plane of section may have a bearing on the observation, but this is not always convincing. The extent and degree of the reaction varies over different areas of the same villus; it culminates in discrete, sometimes very localized, protrusions which may cover a few epithelial cells only, in which the supranuclear cytoplasm of the involved cells appears to be drawn outwards and

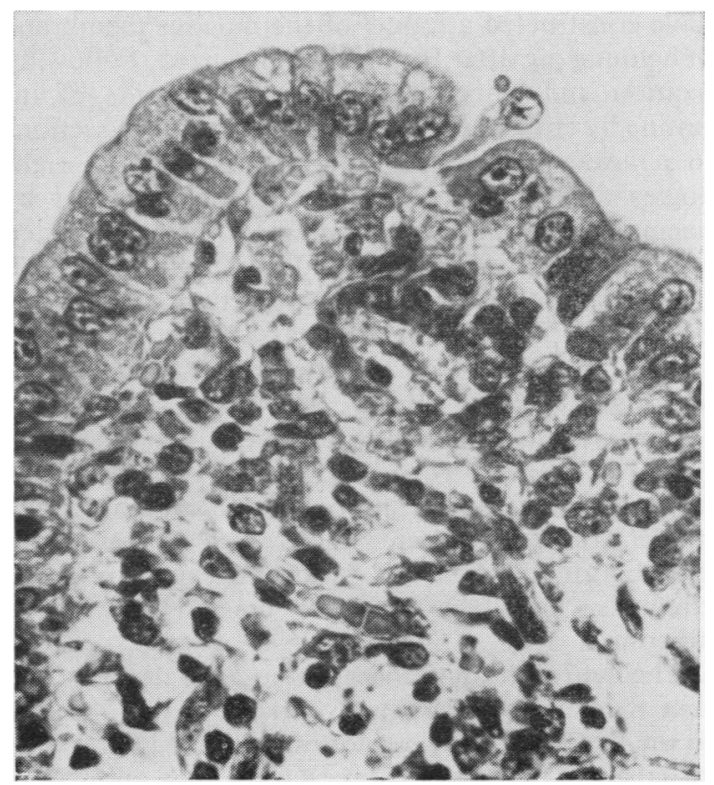

Fig. 7 
capped by the foamy layer described. These protrusions seem to form the adhesive points for adjacent villi, and thereby perhaps to precede the epithelial cell bridges which follow by merging of the contiguous cell cytoplasms (Fig. 5). The frequency of the contact, and perhaps the degree of permanence of the union, could be conditioned by an acute inflammatory response, and the latter could also determine whether fusion of the lamina propria takes place. This also appears to occur only at localized foci, and may be facilitated by the relatively infrequent intense focal infiltration of the epithelium by polymorphonuclear leucocytes.

It should be pointed out that Takeuchi et al (1965) suggest that the frequent fusion of villi in experimental bacillary dysentery was due to excessive loss of intestinal epithelium, with the extruding cells re-attaching to each other, and also bridging intervillous spaces. The author has also seen sections in which the epithelium of one villus appears to have been inserted into a space in the epithelium of another (Fig. 6). That space can be created by loss of cells is clearly shown in Fig. 7 where only one cell appears to be being shed. It is likely that villous fusion is a complex process, dependent upon many factors, and it is of interest in this connexion that if substantiated, the observations described by the author would appear to be initiated by changes in the epithelium, whereas those made by Takeuchi et al (1965) would be secondary to the inflammatory response in the lamina propria.

In order to investigate the effect of fusion, we have constructed a model of the mucous membrane of a young pig after fusions had occurred. Following fixation and embedding of short segments of intestine by conventional techniques 600 serial sections $(6 \mu)$ were cut through the tissue block at right angles to the muscularis mucosa, and stained by haematoxylin and eosin. Each section was projected at a magnification (lateral and horizontal) of $\times 150$ by means of a Projectina projection microscope on to paper $900 \mu$ in thickness. The outline was traced onto the paper, and the profiles were cut out and glued together (Fig. 8). The model showed that the areas of fusion were so complex as to effectively bind all villous structures together, rendering independent movement impossible to envisage. The model also demonstrated that owing to the patchy distribution of areas of fusion, irregular pockets, channels, and burrows were created within the mucosa.

The net result of all these studies is to demonstrate that the mucosa presents a barrier to microbes, or to the metabolic products of microbes, which can be visualized to consist of three layers. The epithelium at its free border is bathed in mucin, which besides

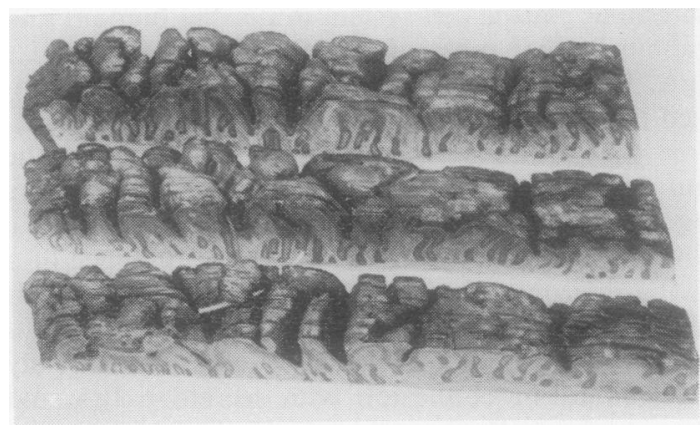

Fig. 8 Reconstruction of the mucosa of a conventional pig. Probes show burrows created beneath the epithelium.

its lysosome content contains secretory immunoglobulins. Secondly, the epithelium can sequester $\vec{\omega}$ microorganisms, but the evidence of Takeuchi et al $\mathrm{O}$ (1965), Takeuchi (1967), and Takeuchi and Sprinz (1967) implies that this might be a deleterious pheno- $\vec{c}$ menon depending on the species of microorganism and its virulence. Finally, the reaction of the lamina propria may be intense and acute as it was in the ${ }^{\gtrless}$ experimental models referred to. At such times $\vec{\theta}$ vascular changes in the lamina precede majof. changes in the epithelium, and the participation phagocytic mechanisms in the defence response becomes marked. The major significance of these observations in clinical terms would seem to relate to any circumstance where, in the normal physiology, biochemistry of the gut was disturbed, eg, by a radical change of diet, and where the small intestinal microbial loading was normally heavy (as in the pig) or pathogens were present in the environment. With such combinations it is likely that all three local defence mechanisms would be involved. Furthermore, where changes in diet and environment are likely to be frequent, the gut will each time pass 3 . through an acute inflammatory response which will $\delta$ be reduced in severity as immunological experience 3 is extended.

\section{References}

Allen, W. D., and Porter, P. (1970). The demonstration of immunoglobulins in porcine intestinal tissue by immunofluorescence with observations on the effect of fixation. Immunology, 18, 799-806.

Ashburner, F. M., and Mushin, R. (1962). Experimental intestinal $\omega$ coliform infections in mice. J. Hyg. (Camb.), 60, 175-181.

Berger, R., Ainbender, E., Hodes, H. L., Zepp, H. D., and Hevizy $<$ M. M. (1967). Demonstration of IgA polioantibody in saliva, duodenal fluid and urine. Nature (Lond.), 214, 420-422.

Brandtzaeg, P. (1968). Glandular secretion of immunoglobulins. $\stackrel{\mathscr{A}}{+}$ Acta path. microbiol. scand., 74, 624.

Burrows, W., Elliott, M. E., and Havens, I. (1947). Immunity to Asiatic cholera. IV excretion of coproantibody in enterio cholera. J. infect. Dis., 81, 261-281. 
Burrows, W., and Havens, I. (1948). Studies on immunity to Asiatic cholera. $\mathrm{V}$ absorption of immune globulin from bowel and its excretion in the urine and feces of experimental animals and human volunteers. J. infect. Dis., 82, 231-250.

Cooper, G. N. (1959). Experimental shigellosis in mice. I. Chronic infection with Shigella dysenteriae type 2. Aust. J. exp. Biol. med. Sci., 37, 193-200.

Crabbé, P. A., Bazin, H., Eyssen, H., and Heremans, J. F. (1968). The normal microbial flora as a major stimulus for proliferation of plasma cells synthesizing IgA in the gut. The germ-free intestinal tract. Int. Arch. Allergy, 34, 362-375.

Crabbé, P. A., Carbonara, A. O., and Heremans, J. F. (1965). The normal human intestinal mucosa as a major source of plasma cells containing $\gamma \mathrm{A}$ immunoglobulin. Lab. Invest., 14, 235-248.

Crandall, R. B., Cebra, J. J., and Crandall, C. A. (1967). The relative proportions of IgG-, IgA- and IgM-containing cells in rabbit tissues during experimental trichinosis. Immunology, 12, 147158.

Davies, A. (1922). Investigation into the serological properties of dysentery stools. Lancet, $2,1009-1012$.

Eidelman, S., and Davis, S. D. (1968). Immunoglobulin content of intestinal mucosal plasma-cells in ataxia telangiectasia. Lancet, $1,884-886$.

Felsenfeld, O., Greer, W. E., and Felsenfeld, A. D. (1967). Cholera toxin neutralization and some cellular sites of immune globulin formation in Cercopithecus aethiops. Nature (Lond.), 213, 12491251.

Freter, R. (1955). Fatal enteric cholera infection in guinea pig, achieved by inhibition of normal enteric flora. J. infect. Dis., 97, 57-65.

Freter, R. (1965). Coproantibody and oral vaccines. In Proceeding of the Cholera Research Symposium, p. 222. Public Health Service Publication 1328, Washington.

Gelzayd, E. A., Kraft, S. C., and Fitch, F. W. (1967). Immunoglobulin A: localization in rectal mucosal epithelial cells. Science, 157, 930-931.

Gelzayd, E. A., Kraft, S. C., and Kirsner, J. B. (1968). Distribution of immunoglobulins in human rectal mucosa. I. Normal control subjects. Gastroenterology, 54, 334-340.

Gordon, H. A. (1968). Is the germ-free animal normal? A review of its anomalies in young and old age. In The Germ-free Animal in Research, edited by Marie E. Coates. Academic Press, London and New York.

Jervis, H. R., Merrill, T. G., and Sprinz, H. (1966). Coccidiosis in the guinea pig small intestine due to a Cryptosporidium. Amer. J. vet. Res., 27, 408-414.
Kenworthy, R. (1967). Intestinal Malabsorption and Diarrhoea in the Newly Weaned Pig. MVSc. Thesis, Liverpool.

Kenworthy, R. (1970). Effect of Escherichia coli on germ-free and gnotobiotic pigs. I. Light and electron microscopy of the small intestine. J. comp. Path., 80, 53-63.

Porter, P., and Kenworthy, R. (1970). Effects of Escherichia coli on germ-free and gnotobiotic pigs. II. Serum proteins and antibodies. J. comp. Path., 80, 233-241.

Porter, P., Noakes, D. E., and Allen, W. D. (1970). Intestinal secretion of immunoglobulins and antibodies to Escherichia coli in the pig. Immunology, 18, 909-920.

Rubin, W., Fauci, A. S., Sleisenger, M. H., and Jeffries, G. H. (1965). Immunofluorescent studies in adult coeliac disease. $J$. clin. Invest., 44, 475-485.

Smith, H. W. (1965). Observations on the flora of the alimentary tract of animals and factors affecting its composition. J. Path. Bact., 89, 95-122.

Sojka, W. J. (1965). Escherichia coli in Domestic Animals and Poultry. (Review Series no. 7 of the Commonwealth Bureau of Animal Health, Weybridge.) Commonwealth Agricultural Bureaux, Farnham Royal, Bucks, England.

Sprinz, H., Kundel, D. W., Dammin, G. J., Horowitz, R. E., Schneider H., and Formal, S. B. (1961). The response of the germ-free guinea pig to oral bacterial challenge with Escherichia coli and Shigella flexneri. Amer. J. Path., 39, 681-695.

Stobo, J. D., and Tomasi, T. B., Jr. (1967). A low molecular weight immunoglobulin antigenically related to 19 S IgM. J. clin. Invest., 46, 1329-1337.

Takeuchi, A. (1967). Electron microscope studies of experimental Salmonella infection. I. Penetration into the intestinal epithelium by Salmonella typhimurium. Amer. J. Path., 50, 109-136.

Takeuchi, A., and Sprinz, H. (1967). Electron-microscope studies of experimental Salmonella infection in the preconditioned guinea pig. II. Response of the intestinal mucosa to the invasion by Salmonella typhimurium. Amer. J. Path., 51, 137-162.

Takeuchi, A., Sprinz, H., LaBrec, E. H., and Formal, S. B. (1965). Experimental bacillary dysentery. An electron microscopic study of the response of the intestinal mucosa to bacterial invasion. Amer. J. Path., 47, 1011-1044.

Tomasi, T. B. (1967). The gamma A globulins: first line of defence. Hosp. Pract., 2, 26. 